\title{
Optical observation of single atomic ions interacting with plasmonic nanorods in aqueous solution
}

\author{
Martin D. Baaske ${ }^{1}$ and Frank Vollmer ${ }^{1 *}$ \\ ${ }^{1}$ Max Planck Institute for the Science of Light, \\ Günther-Scharowsky-Straße 1, 91058 Erlangen, Germany
}

\begin{abstract}
Plasmonic nanoparticles provide the basis for a multitude of applications in chemistry, health care, and optics due to their unique and tunable properties. Nanoparticle based techniques have evolved into powerful tools for studying molecules and their specific interactions even at the single molecule level. Here we show that this sensing capability can be used for detecting single atomic ions in aqueous medium. We monitor interactions of single zinc and mercury ions with plasmonic gold nanorods resonantly coupled to our whispering gallery mode sensor. Our system's ability to discern permanent binding and transient interaction allows us to study ion specific interaction kinetics. Saturation free detection of single ions in the transient interaction regime enables us to statistically confirm that the sensor signals originate from single ions. Furthermore, we reveal how the ion nanorod interactions evolve with respect to the medium's ionic strength as mercury ions amalgamate with gold and zinc ions eventually turn into probes of highly localized surface potentials. Therefore this study might lay the cornerstone for the optical investigation of atomic processes at nanoparticle surfaces and in liquid medium.
\end{abstract}


Plasmonic nanoparticles (NPs), have been used for a wide range of applications such as spectroscopy [1, 2], high resolution imaging [3], medical therapy [4-6], nonlinear optics [7] and photocatalysis $[8,9]$. Such widespread utility is attributable to the diverse range of NP shapes and sizes available and the corresponding tunability of their optical properties [10-14]. The highly localized fields near the surface of such particles, in addition to their small mode volumes, makes them especially suitable for sensing $[15,16]$ or even capable of label-free single molecule detection [17-19]. The NPs' small surface area, however, imposes a severe restriction on these sensor systems since, upon binding, each individual molecule occupies an additional fraction of the NP's surface, finally rendering it insensitive once full coverage is reached. Systems operating in the binding regime thus usually require use of multiple NPs in order to obtain statistically significant datasets. Recently, we introduced a technique capable of mitigating this disadvantage by detecting single analyte molecules in the transient regime whereby no depletion via permanent binding of analyte occurs [20]. We achieved single molecule sensitivity by resonantly coupling nanorods (NR) to whispering gallery mode (WGM) microresonators, which by themselves are sensitive sensors [21, 22], capable of detecting single nanoparticles and viruses [23-26], and bulk recognition of small molecules [27-29] and heavy metal ions [30,31]. In this work we employ our platform for detecting the interaction of single mercury and zinc ions with gold NRs in an aqueous medium. Toxic mercury is a major environmental hazard [32, 33] and zinc plays a crucial role in a plethora of biological processes [34,35], such that this work demonstrates the utility of our technique for environmental monitoring and sensing. Performing the experiment under conditions allowing only for transient ion-NR interactions enables us to statistically confirm the single ion nature of the observed signals independently from the total number of available sensing volumes. Furthermore we elaborate on the effects arising from the presence of additional electrolytes since they can give rise to mercury binding or transitions in the transient behavior of zinc ions. This establishes our sensor platform as a tool for monitoring analyte NR reactions on an atomic level, as well as opening up a new way to study processes in the electric double layer on a single ion basis. 

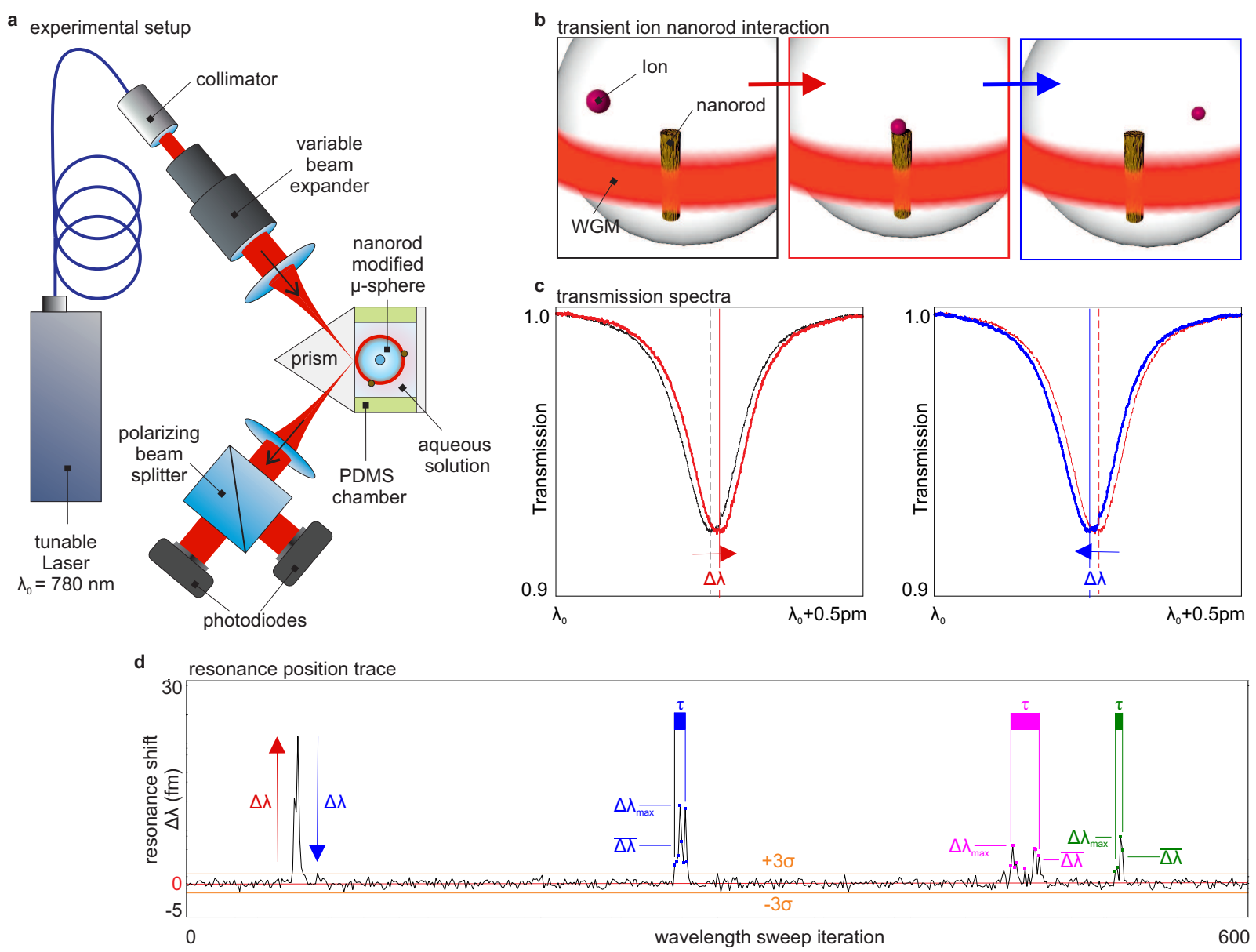

FIG. 1. Experimental method and extraction of transient events. a, Layout of the WGM sensing setup. Light from a wavelength tunable laser is evanescently coupled into a NR modified microsphere via frustrated total internal reflection at a prism's surface. Transient interactions of single zinc and mercury ions with the NRs (b), excited at their plasmon resonance, are detected as a red shift of the WGM resonance (c, left) when an ion enters a sensing sites on a NR's surface, and a subsequent blue shift (c, right) when the ion leaves the sensing site. Transient ion NR interactions are observed as distinct spikes in traces of the resonance positions (d). The trace is processed by the algorithm described in suppl. sect. 1C in order to determine the maximum $\left(\Delta \lambda_{\max }\right)$ and mean shift $(\overline{\Delta \lambda})$ values as well as the length $(\tau)$ of each spike (here due to $\mathrm{Hg}$ NR interactions) exceeding $3 \sigma$. 
The single ion detection experiments were carried out using our robust prism coupling platform [20]. We excite the WGMs of NR modified fused silica microspheres by frustrated total internal reflection of a laser beam focused onto a prism's surface (Fig. 1a). Resonantly excited high Q modes are observed as dips of Lorentzian shape in the transmission spectrum (Fig. 1c), which is obtained by sweeping the wavelength of a tunable external cavity laser (center wavelength $780 \mathrm{~nm}$ ). The WGM's evanescent field resonantly excites the NR's localized surface plasmon and thus couples the microresonator with the sensing sites on the NR's surface. These sensing sites are related to local intensity hot spots and thus they are mostly at the tips of the NRs. When a zinc or mercury ion enters the sensitive sites and interacts with the NR (Fig. 1b) the coupling between the NR and microcavity transduces this interaction into a red shift $(\Delta \lambda)$ of the WGM's resonance position (Fig. 1c, left). For a permanent interaction, such as formation of a strong covalent bond, the resonance will remain in this shifted position, however, when an ion interacts with a NR in a way such that it only remains confined in a sensing site for a certain period of time $\tau$, the resonance position is shifted back towards its original position after the ion leaves the sensing site (Fig. 1c, right). As we trace the WGM position over time, permanent interactions manifest as a step pattern (Fig. 4a), whereas transient interactions appear as fast changes exhibiting a spike pattern (Fig. 1d). We have developed an algorithm, described in section 1B of the supplementary information, which allows for the extraction of these spikes in contrast to the slow drift of the resonance position caused by changes in ambient conditions such as temperature or pressure. The removal of these background drifts from the original traces allows us to determine the values of the maximum $\Delta \lambda_{\max }$ and mean resonance shifts $\overline{\Delta \lambda}$ as well as the length $\tau$ of individual spike events. Events are identified based on deviations from the drift free trace with a magnitude greater than $3 \sigma$, where $\sigma$ is the standard deviation of the noise of the drift free trace (Fig. 1d). In the following we will use these transient interaction characteristics to statistically prove that the spikes are caused by single interactions, as well as to compare $\mathrm{Hg} \mathrm{NR}$ and Zn NR interactions. 

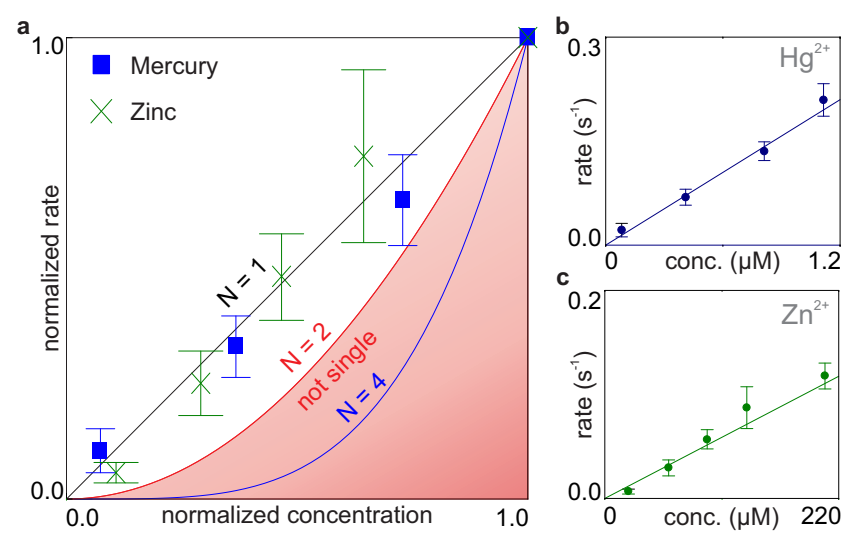

FIG. 2. Dependence of spike rates on analyte concentration. a, Comparison of experimentally obtained concentration dependent spike detection rates (points) for zinc and mercury ions (normalized to the respective maximum values) with the dependence expected if a minimum number $(\mathrm{N})$ of 1,2 , or 4 interactions are necessary to detect one spike event (lines). Panel $\mathbf{b}$, and (c) show the absolute values of these rates (points), as obtained with different NR modified microspheres, and corresponding linear fits for mercury and zinc ions, respectively. Error bars on rates represent their standard deviation based on Poissonian statistics.

\section{Statistic properties of transient single ion interactions}

Our system's capability to detect single ions relies on sensing sites found on the NRs coupled to the cavity, the number of which is unknown and differs between individual experiments. The limited number of sensing sites, and hence the potential of saturation and loss of sensitivity, can be mitigated by operating the sensor in conditions which allow only for transient analyte NR interactions thus maintaining a constant number of sensing sites. Sensor operation in this depletion free regime enables us to perform and compare a series of experiments in which we vary the parameters, e.g. the solution's $\mathrm{pH}$, the concentration of analyte ions, or the concentration of additional electrolytes, with a single NR modified microsphere. We exploit this advantage to statistically confirm the single interaction nature of the detected spike events. Under the assumption that our system is not able to discern whether a spike is caused by one or more interactions, and that our detection process is governed by an underlying Poisson process, we have derived a scaling law for the concentration 
dependent spike rate (see supplement section 3). From this we can make the approximation

$$
\frac{R\left(m \cdot c_{0}\right)}{R\left(c_{0}\right)} \approx m^{N},
$$

where $R(c)$ is the concentration dependent spike rate, $c_{0}$ is a reference concentration, $N$ denotes the minimum number of interactions necessary to cause a spike event, and $m$ is the ratio of the actual and reference concentrations. Thus we expect the rate of events to depend linearly on analyte concentration if the spikes originate from single interactions $(N=1)$. As evident from Fig. 2a our experimentally obtained rates match this expectation for both $\mathrm{Zn}$ and $\mathrm{Hg}$ ions. Specifically, all observed rates match the theoretical curve for $N=1$ inside their errors with one exception, which nevertheless still lies above the curve for $N=2$. Furthermore the experimentally obtained probability distributions are in good agreement with the corresponding Poisson distributions (Supp. fig. S7) and the rates obtained by fitting these distributions show good agreement with the respective directly extracted rates i.e., the ratio of the total event count over the time between the first and the last event, within the respective errors (Supp. table S2). This therefore confirms the assumption that our detection is based on a Poisson process. Moreover, this observation together with the linear dependence of the rate on the concentration, demonstrates the observed spike events originate from only a single interaction.

Although both $\mathrm{Hg}$ and $\mathrm{Zn}$ ions exhibited similar behavior with regards to their normalized rate, inspection of the absolute values of the concentration dependent rates (Fig. 2b, c) reveals a significant difference. Specifically the rate constants differ and are found to be $(0.53 \pm 0.05) \times 10^{3}(\mathrm{Ms})^{-1}$ for zinc ions, and $(175 \pm 7) \times 10^{3}(\mathrm{Ms})^{-1}$ for mercury ions. This disparity most likely arises from an overall difference in the available number of sensing sites as the measurement series were performed with different NR modified microcavities. The similar spike magnitude and length distributions found for both types of ions, however, indicate similar levels of sensitivity (Fig. 3a). In general the recorded wavelength shifts are well in excess of $3 \sigma$, where $\sigma$ typically ranges from 0.5 to $0.7 \mathrm{fm}$. The overall average measured $\overline{\Delta \lambda}, \Delta \lambda_{\max }$ and $\tau$ values were, $4.9 \mathrm{fm}, 9.6 \mathrm{fm}$ and $0.27 \mathrm{~s}$ for mercury and $4.4 \mathrm{fm}, 6.6$ $\mathrm{fm}$ and $0.36 \mathrm{~s}$ for zinc ions. A significant fraction of the observed spikes, namely $28.4 \%$ of all mercury and $38.8 \%$ of all zinc events, had a length, $\tau$, of $19 \mathrm{~ms}$, the time it takes to perform a complete wavelength sweep of the exciting laser. These events require further investigation so as to determine whether it is necessary for an interaction to be as long as one scanning 

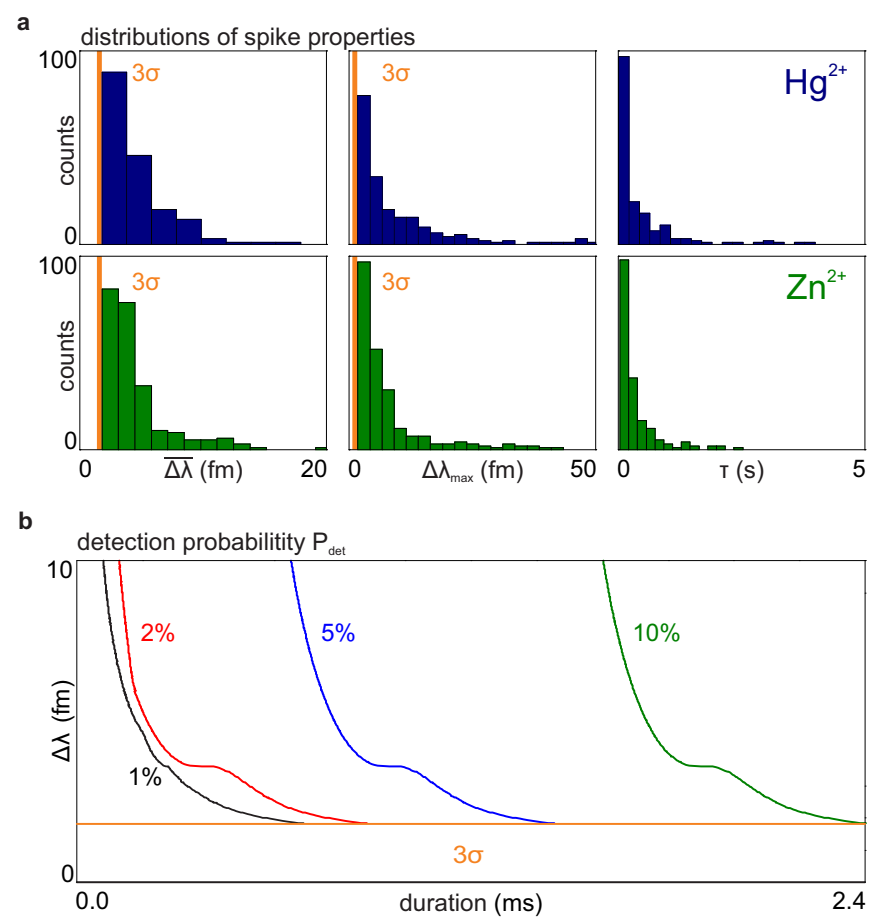

FIG. 3. Statistics of spikes properties and theoretical detection probabilities for short interactions. a, Example distributions of average and maximum spike shifts and spike durations obtained during $\approx 30$ minutes. $\mathbf{b}$, Curves of constant detection probabilities of $1,2,5$, and $10 \%$ simulated for interactions shorter than one scanning period, depending on shift size and event duration. The shoulder like features are artifacts caused by the centroid method used to determine the resonance positions.

period in order to be recognized or whether our system is capable of resolving shorter interactions. To estimate the actual lower limits of the experimental time resolution we have performed mode distortion simulations based on our average experimental parameters, namely a WGM linewidth of $224 \mathrm{fm}$ corresponding to a cavity Q-factor of $4.4 \times 10^{6}$, a noise floor with a standard deviation of $\sigma=0.6 \mathrm{fm}$, and a laser scanning range of $9.75 \mathrm{pm}$. The details of the simulations are given in section 2 of the supplement. Based on these simulations we can provide an estimate for $P_{d e t}$, the probability of detecting a resonance shift with a certain magnitude and duration, as a single point spike in excess of $3 \sigma$. The resulting estimates for the detection probabilities are displayed in Fig. 3b in the form of lines of constant probability. We find that even events with durations up to a hundred times shorter than the scanning period can be detected albeit with a rather low probability. 
Detection occurs when a short event coincides with the excitation period of a WGM which accounts for only a short interval, here $\approx 0.4 \mathrm{~ms}$, of a complete scanning period. It is important to note that the resulting time resolution $(\approx 0.1 \mathrm{~ms})$ is not sufficient for detection of freely diffusing ions in water as they have diffusion constants of $7.03 \times 10^{-6} \mathrm{~cm}^{2} \mathrm{~s}^{-1}\left(\mathrm{Zn}^{2+}\right)$ and $9.13 \times 10^{-6} \mathrm{~cm}^{2} \mathrm{~s}^{-1}\left(\mathrm{Hg}^{2+}\right)[36]$ and therefore their root mean squared displacements for periods of $0.1 \mathrm{~ms}$ are in the range of 0.6 to 0.8 micrometers, which greatly exceeds the dimensions of the NRs. This also implies that an attractive force between the ions and NRs is required in order to confine the ions long enough inside the sensing sites to allow for their detection. Furthermore the detection of spike events from $\mathrm{Zn}$ and $\mathrm{Hg}$ ions, as discussed above, is only possible in solutions with $\mathrm{pH} 7$ and an ionic strength on the order of $1 \mathrm{mM}$. The latter was controlled by the addition of sodium chloride or magnesium perchlorate in the case of zinc and mercury, respectively. These additional electrolyte ions, however, did not cause identifiable spike or step events during our control experiments (suppl. sect. 4). This supports the assumption that these additional ions influence the electrostatic force between the analyte ions and the NRs and thus the $\mathrm{Hg}$, Zn NR interaction. In what follows, we will therefore investigate the effect of the electrolyte concentration on the behavior of the ion NR interaction further.

\section{Evolution of the ion nanorod interaction with increasing ionic strength}

We investigate the influence of the electrolyte ionic strength of the aqueous solution on the ion-NR-interaction by increasing it stepwise while preserving a constant concentration of analyte ions. We do not observe a significant influence of the ionic strength, as set by magnesium perchlorate and sodium chloride for zinc and mercury respectively, below values of $14.6 \mathrm{mM}$ in the case of mercury and $18 \mathrm{mM}$ in the case of zinc as the length, magnitude and rate of spike events remain almost constant. By increasing the ionic strength beyond these values, however, we find a fundamental difference in the way both species of analyte ions interact with the gold NRs. In what follows, we therefore discuss both ion species separately, initially focusing on the evolution of the interaction of Hg. As shown in the traces in Fig. 4a both spike and step transitions in the resonance position are observed for an ionic strength of $14.6 \mathrm{mM}$, whereas at an even higher ionic strength of $60 \mathrm{mM}$ step type transitions occur predominantly. These abrupt but permanent shifts in resonance position 


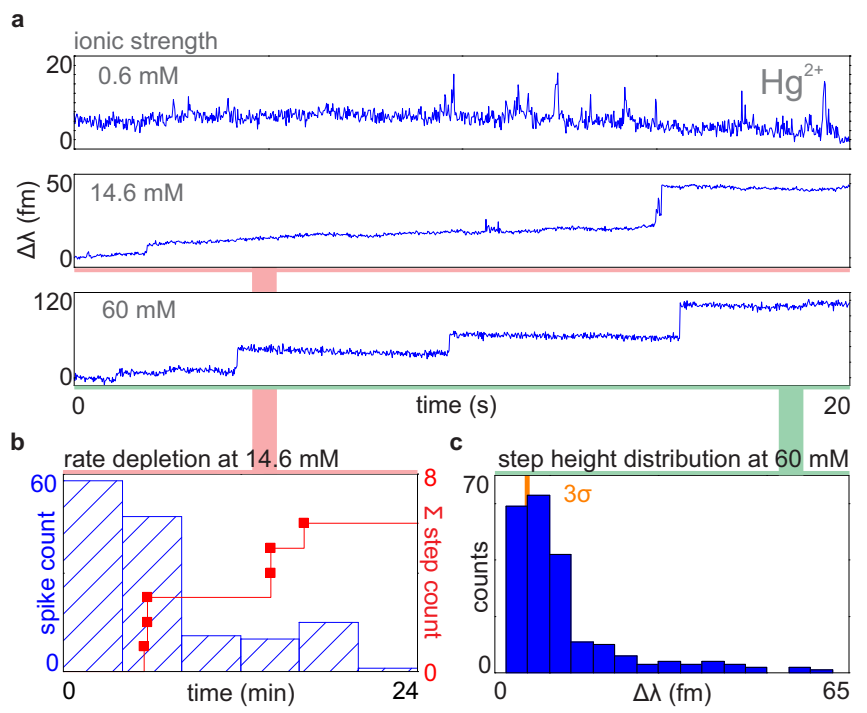

FIG. 4. Ionic strength dependence of the mercury nanorod interaction. a, WGM resonance wavelength traces displaying the transition from only spike events at $0.6 \mathrm{mM}$ ionic strength (top) to step events at $60 \mathrm{mM}$ ionic strength (bottom). Both types of events can be observed in the middle trace obtained at a ionic strength of $14.6 \mathrm{mM}$. b. Comparison between the number of spikes in constant 4 min intervals (blue) and the cumulative step count (red) obtained simultaneously at an ionic strength of $14.6 \mathrm{mM}$. c, Histogram showing the distribution of step magnitudes obtained with a different NR modified microsphere and an ionic strength of $60 \mathrm{mM}$.

indicate the formation of stable bonds between the mercury ions and the NRs. Since we do not observe any step transitions towards shorter wavelengths, as would be expected upon the breaking of these bonds, the formation of these bonds can be assumed to be an irreversible process. It has been reported that mercury ions can be detected in aqueous solution with the use of gold NRs due to the amalgamisation of gold with mercury in the presence of the reducing agent, sodium borohydride [37]. With no such reducing agent present in our experiments, the binding process we observe might be due to the reduction of mercury ions by light induced hot carriers created in the NRs themselves followed by the amalgamisation, as a similar light induced process has previously been reported to account for the efficient reduction of silver ions [38]. Apart from this interesting finding, which possibly implies the optical observation of a single atom as it forms a chemical bond with the atoms of a nanoparticle, we can gain further information about the sensing sites on the NRs by studying the interactions in the ionic strength regime where both, spike and step, 
events are recognized. In figure $4 \mathrm{~b}$ we show the number of spikes observed during intervals with a constant length of 4 minutes and the cumulative count of steps recognized during the course of the same experiment. The graph shows a clear decrease in the rate of spike recognition as the number of observed steps increases. This indicates that once a sensing site is occupied by binding of an ion, it is either blocked or loses its sensitivity for further transient interactions. Nonetheless, we can conclude that the sites on the NRs which are sensitive to transient interactions are identical to the ones sensitive to binding type interactions. The similarity of the step magnitude (Fig. 4c) and the maximum shift distributions (Fig. 3a) further supports this conclusion. In case of the zinc ions, however, the recorded WGM traces

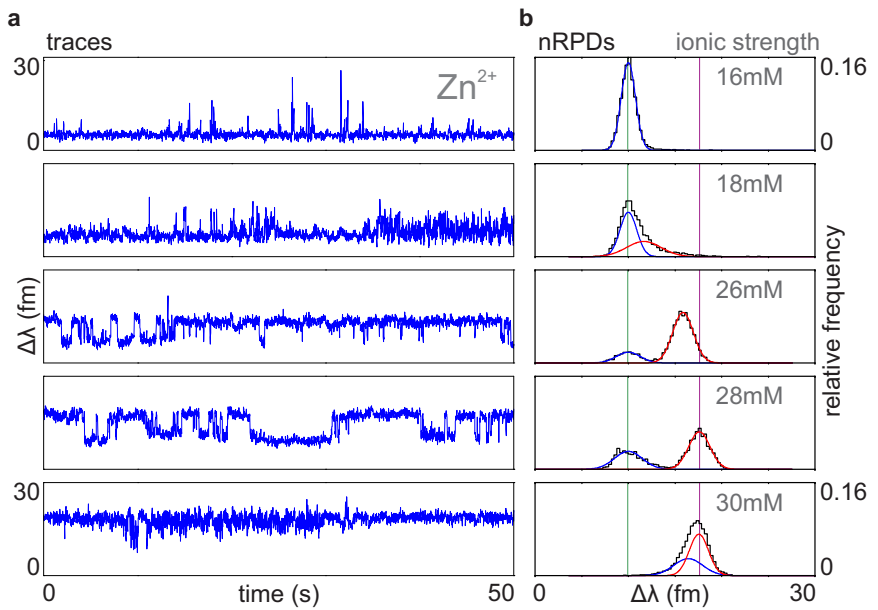

FIG. 5. Ionic strength dependence of the zinc ion nanorod interaction. The panels in a display example wavelength traces, with the slow varying background removed, obtained at increasing ionic strength and constant zinc concentration of $8 \mu \mathrm{M}$. The corresponding distributions (black) of the wavelength positions extracted from the whole traces are displayed in $\mathbf{b}$ together with the fitted Gaussian distributions (blue, red). The vertical green line indicates the unperturbed ground state and the vertical purple line the maximum observed perturbed state.

do not provide any indications of the formation of irreversible bonds between ions and NRs. Instead we find the transient interaction to evolve through different states as we increase the ionic strength. Example traces recorded with a single NR-modified microsphere showing these different states are shown in figure 5 a alongside their respective normalized resonance position distributions (nRPD) in Fig. 4b. Starting at an ionic strength of $16 \mathrm{mM}$, as set with sodium chloride, we observe exclusively spike events and thus the nRPD shows a Gaussian peak representing the noise floor of the unperturbed WGM with a few outliers due to the 
spikes. Upon increasing the ionic strength to $18 \mathrm{mM}$, however, we observe an additional type of transient events, which can be described as extended intervals during which the resonance trace exhibits an increased noise amplitude. These intervals have abrupt beginnings and ends and can last for periods in excess of 30 seconds. In the nRPDs these bursts show up as an asymmetric extension of the Gaussian peak towards the longer wavelength side. As the ionic strength reaches values between $26 \mathrm{mM}$ to $28 \mathrm{mM}$ the temporal characteristic of the ion $\mathrm{NR}$ interaction transforms again to what can be described as sudden jumps between two states interspersed by intervals of different length. The corresponding nRPDs for these cases show two clearly separated Gaussian distributions. This discrete behavior indicates a relatively long and stable but reversible adsorption of a zinc ion to a NR sensing site. As we only observed two distinct levels in the nRPDs this type of interaction was most likely limited to a single sensing site. While this does not imply the existence of only one sensing site, as we observe additional spikes superimposed on the two level trace (middle trace in Fig 5a), it does imply that different sensing sites on the NRs might support interactions on different timescales. From this we conclude that the duration of transient zinc ion interactions is strongly influenced by the local environment of each sensing site. As we increase the ionic strength even further to $30 \mathrm{mM}$ we again observe spike and burst type events but with opposite sign since the shifts in the resonance position occur towards the shorter wavelength side of the spectra. The ions therefore remain mostly confined inside the sensing site under these conditions although they are still prone to local environmental fluctuations which can cause relatively small transient changes in their position with respect to the NR's surface.

\section{Model of the interaction and discussion}

We have so far reported on how the ionic strength of the surrounding medium influences the ion NR interaction. To further understand the physical origin of the different types of observed interactions, in the following we will outline a possible model for the underlying potential governing the interaction. Illustrations representing the different states of the model system are shown in figure 6 (left) along with sketches of the potential energy governing the interaction and representative measured traces (right) for each case. The NRs used are capped with bilayers of cethyltriammonium bromide (CTAB) which allow the particles to obtain the rod shape during their growth as it covers the cylindrical surface more densely 

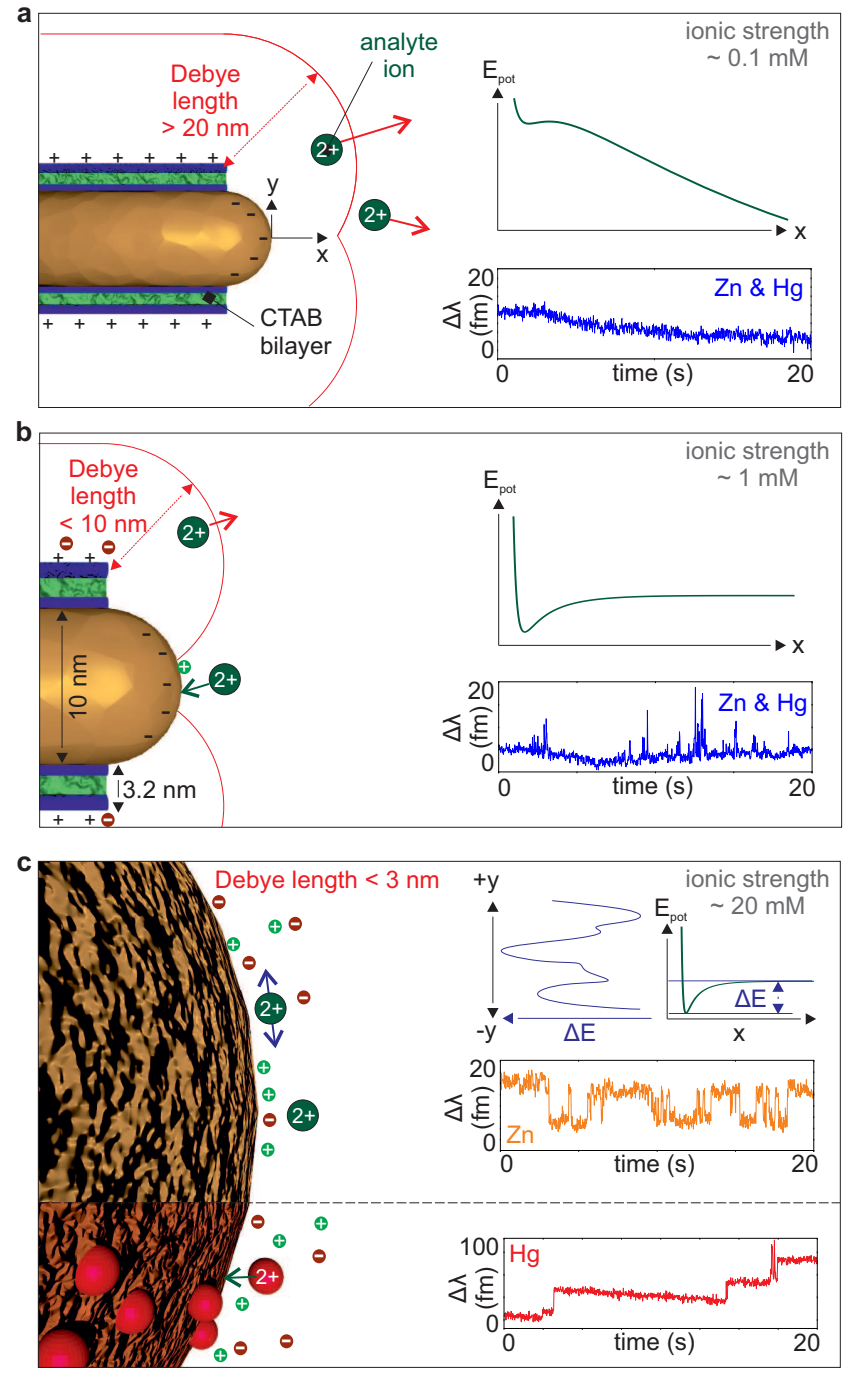

FIG. 6. Model of three interaction regimes Panels from left to right illustrate the system at the scale of the corresponding Debye length, sketches of the analyte ions potential energy and example experimental wavelength traces. a, At low ionic strength the repulsive potential caused by the positively charged CTAB prevents ion-NR interactions and no spikes or steps are observed. b, Above a $1 \mathrm{mM}$ ionic strength Debye screening of the CTAB layer allows the analyte ions to be temporarily confined by the attractive potential created by the NR's negative surface charge and spikes are observed. c, At high ionic strengths the Zn ions remain confined longer in the local minima of potential energy found along the NR's surface and the $\mathrm{Hg}$ ions bond permanently to the NRs. Step transitions towards the red and the blue side of the spectrum are observed for Zn, and towards the red for $\mathrm{Hg}$. 
than the spherical surface at both ends of the NR [13]. As this surface layer carries a positive charge it creates a repulsive potential for the positively charged zinc and mercury ions effectively preventing them from reaching the sensing sites located at both ends of the NR (Fig.6a). Upon introduction of additional electrolyte into the system, the negatively charged ions (chloride or perchlorate) start to form a layer around the positively charged end groups of the CTAB bilayer. The resulting Debye screening reduces the repulsive force seen by the analyte ions consequently allowing them to get closer to the tips of the NRs which are less densely covered with CTAB. We assume that the tips of the NRs carry a negative surface charge since the solution's $\mathrm{pH}$ is above the isoelectric point of gold [39, 40]. This surface charge, while also screened by counterions, provides for a short ranged attractive potential, which is sufficiently deep to allow for trapping the mercury and zinc ions long enough to be detected before they thermally obtain the kinetic energy required to escape (Fig.6b). These assumptions are supported by our experiments as we start seeing spike type events at an ionic strength of about $1 \mathrm{mM}$ corresponding to a Debye length of approximately $9.5 \mathrm{~nm}$ a value slightly smaller than minimum distance between the CTAB endgroups on the cylindrical surface of the NR and the tip of the NR $(11 \mathrm{~nm})$. For an ionic strength in the range of 15 to $20 \mathrm{mM}$ we observed the onset of burst type and binding type interactions for zinc and mercury ions, respectively (Fig. 6c). At these values the Debye length is shorter than $2.5 \mathrm{~nm}$ thus the influence of the charges carried by the endgroups of the $3.2 \mathrm{~nm}$ thick CTAB bilayer $[41,42]$ on ions localized at the NR's surface is negligible. At this ionic strength zinc ions might therefore act as probes of the local charge density on the NRs surface as they are eventually confined in areas with higher charge densities which originate from edge like surface features or impurities on an atomic scale as sketched in Fig. 6c.

\section{Conclusions}

We have experimentally demonstrated and statistically confirmed the optical detection of single mercury and zinc ions upon their interaction with gold nanorods. We have studied the influence of the solution's ionic strength on the ion NR interaction for both kinds of ions and found a clear difference in their behavior. Mercury ions are found to form permanent bonds with the gold NRs whereas zinc ions are confined by local attractive surface potentials on the NRs. Based on our experimental results we have derived a simple electrostatic model 
to explain the different types of transient interactions. Furthermore, we have demonstrated that the ability of our sensor system to operate under conditions allowing only for transient ion NR interactions and thus, free of depletion effects, makes our system a powerful tool for statistical analysis even, and especially, when only a few sensing sites are available. This study therefore lays the foundation for the optical investigation of atomic processes on the surface of plasmonic particles. Our method holds not only the prospect of gaining further insight into processes occurring inside the electric double layer or the atom by atom observation of nanoparticle growth but might also be applicable for studying biological systems, such as ion channels or metalloenzymes, or catalytic effects of metal surfaces on chemical reactions on an atomic level.

\section{Methods}

Chemicals were obtained from Sigma-Aldrich and Carl Roth. All solutions were made from ultrapure water obtained from VWR. Solutions containing zinc and mercury ions were made with zinc nitrate and mercury nitrate. All solutions except the ones containing NRs were filtered with $0.1 \mu \mathrm{m}$ membrane filters (Merck Millipore) prior to measurements.

Fused silica microspheres with diameters between 70 and $90 \mu \mathrm{m}$ were fabricated from SMF-28 standard telecommunication fibers (Dow Corning) by melting using a $\mathrm{CO}_{2}$-Laser.

The solutions are injected into interchangable U-shaped polydimethylsiloxane (PDMS) chambers, that are pressed against the prism, able of holding volumes in the range from 0.5 to 0.7 milliliters.

The CTAB capped gold NRs (Diameter: $10 \mathrm{~nm}$, length $42 \mathrm{~nm}$, Nanopartz Inc.), with a surface plasmon resonance at a wavelength of $780 \mathrm{~nm}$, are immobilized onto the microresonator in aqueous solution containing perchloric acid with a concentration of $24 \mathrm{mM}$. During this deposition process the binding of NRs to the resonator surface is recognized as distinct changes in resonance position $\lambda$ and linewidth $\gamma$. The deposition process is stopped by evacuating the chamber after observation of at least one NR binding event which causes the linewidth to broaden by $\Delta \gamma \geq 20 \mathrm{fm}$ to a total of not more than $\gamma=200 \mathrm{fm}$ thus maintaining a resonance quality factor in excess of $4 \times 10^{6}$. The chamber is subsequently rinsed with water before ion detection experiments are performed.

The extraction of linewidth and the resonance position from measured spectra is done 
with the fixed threshold centroid method (for details please see suppl. sect. 1A) .

Wavelength tunable laser: Toptica Dl pro with a center wavelength of $780 \mathrm{~nm}$. Prism material : N-SF 11.

\section{Acknowledgments}

The authors acknowledge financial support for this work from the Max Planck Society. M.D.B. thanks M.R. Foreman and E. Kim for their feedback on the manuscript.

\section{Author contributions}

M.D.B. developed the experimental setup, performed the experiments and data analysis and wrote the manuscript. F.V. commented on the manuscript and supervised the project.

\section{Additional information}

The authors declare no competing financial interests.

[1] Jackson, J. B. \& Halas, N. J. Surface-enhanced Raman scattering on tunable plasmonic nanoparticle substrates. Proceedings of the National Academy of Sciences of the United States of America 101, 17930-17935 (2004).

[2] Zamarion, V. M., Timm, R. A., Araki, K. \& Toma, H. E. Ultrasensitive SERS nanoprobes for hazardous metal ions based on trimercaptotriazine-modified gold nanoparticles. Inorganic Chemistry 47, 2934-2936 (2008).

[3] Sonnefraud, Y. et al. Experimental proof of concept of nanoparticle assisted STED. Nano Letters 14, 4449-4453 (2014).

[4] Huang, X., El-Sayed, I. H., Qian, W. \& El-Sayed, M. A. Cancer cell imaging and photothermal therapy in the near-infrared region by using gold nanorods. Journal of the American Chemical Society 128, 2115-2120 (2006). 
[5] El-Sayed, I. H., Huang, X. \& El-Sayed, M. A. Selective laser photo-thermal therapy of epithelial carcinoma using anti-EGFR antibody conjugated gold nanoparticles. Cancer Letters 239, 129-135 (2006).

[6] Han, G., Ghosh, P. \& Rotello, V. M. Functionalized gold nanoparticles for drug delivery. Nanomedicine 2, 113-123 (2007).

[7] Kauranen, M. \& Zayats, A. V. Nonlinear plasmonics. Nature Photonics 6, 737-748 (2012).

[8] Mukherjee, S. et al. Hot Electrons Do the Impossible: Plasmon-Induced Dissociation of $\mathrm{H}_{2}$ on Au. Nano Letters 13, 240-247 (2012).

[9] Wang, F. et al. Plasmonic Harvesting of Light Energy for Suzuki Coupling Reactions. Journal of the American Chemical Society 135, 5588-5601 (2013).

[10] Frens, G. Controlled Nucleation for the Regulation of the Particle Size in Monodisperse Gold Suspensions. Nature Physical Science 20-22 (1973).

[11] Ha, T. H., Koo, H.-j. \& Chung, B. H. Shape Controlled Syntheses of Gold Nanoprism and Nanorod Influenced by Specific Adsorption of Halide Ions. J. Phys. Chem. C 111, 1123-1130 (2007).

[12] Busbee, B. D., Obare, S. O. \& Murphy, C. J. An improved synthesis of high-aspect-ratio gold nanorods. Advanced Materials 15, 414-416 (2003).

[13] Nikoobakht, B. \& El-Sayed, M. A. Preparation and Growth Mechanism of Gold Nanorods (NRs) Using Seed - Mediated Growth Method. Chemistry of Materials 15, 1957-1962 (2003).

[14] Kumar, P. S., Pastoriza-Santos, I., Rodriguez-Gonzalez, B., Javier Garcia de Abajo, F. \& LizMarzan, L. M. High-yield synthesis and optical response of gold nanostars. Nanotechnology 19, 015606 (2008).

[15] Haes, A. J., Chang, L., Klein, W. L. \& Van Duyne, R. P. Detection of a biomarker for Alzheimer's disease from synthetic and clinical samples using a nanoscale optical biosensor. Journal of the American Chemical Society 127, 2264-2271 (2005).

[16] Anker, J. N. et al. Biosensing with plasmonic nanosensors. Nature Materials 7, 442-453 (2008).

[17] Ament, I., Prasad, J., Henkel, A., Schmachtel, S. \& Sönnichsen, C. Single unlabeled protein detection on individual plasmonic nanoparticles. Nano Letters 12, 1092-1095 (2012).

[18] Zijlstra, P., Paulo, P. M. R. \& Orrit, M. Optical detection of single non-absorbing molecules using the surface plasmon resonance of a gold nanorod. Nature Nanotechnology 7, 379-382 
(2012).

[19] Beuwer, M. A., Prins, M. W. J. \& Zijlstra, P. Stochastic protein interactions monitored by hundreds of single-molecule plasmonic biosensors. Nano Letters 15, 3507-3511 (2015).

[20] Baaske, M. D., Foreman, M. R. \& Vollmer, F. Single-molecule nucleic acid interactions monitored on a label-free microcavity biosensor platform. Nature Nanotechnology 9, 933-939 (2014).

[21] Baaske, M. \& Vollmer, F. Optical resonator biosensors: Molecular diagnostic and nanoparticle detection on an integrated platform. ChemPhysChem 13, 427-436 (2012).

[22] Foreman, M., Swaim, J. \& Vollmer, F. Whispering gallery mode sensors. Advances in Optics and Photonics 7, 168-168 (2015).

[23] Vollmer, F., Arnold, S. \& Keng, D. Single virus detection from the reactive shift of a whispering-gallery mode. Proceedings of the National Academy of Sciences of the United States of America 105, 20701-4 (2008).

[24] He, L., Ozdemir, S. K., Zhu, J., Kim, W. \& Yang, L. Detecting single viruses and nanoparticles using whispering gallery microlasers. Nature Nanotechnology 6, 428-432 (2011). 1107.0868.

[25] Lu, T. et al. High sensitivity nanoparticle detection using optical microcavities. Proceedings of the National Academy of Sciences of the United States of America 108, 5976-5979 (2011).

[26] Foreman, M. R., Jin, W.-L. \& Vollmer, F. Optimizing detection limits in whispering gallery mode biosensing. Optics Express 22, 5491-511 (2014).

[27] Vollmer, F. et al. Protein detection by optical shift of a resonant microcavity. Applied Physics Letters 80, 4057-4059 (2002).

[28] Washburn, A. L., Gunn, L. C. \& Bailey, R. C. Label-free quantitation of a cancer biomarker in complex media using silicon photonic microring resonators. Analytical Chemistry 81, 9499$9506(2009)$.

[29] Wu, Y., Zhang, D. Y., Yin, P. \& Vollmer, F. Ultraspecific and highly sensitive nucleic acid detection by integrating a DNA catalytic network with a label-free microcavity. Small 10, 2067-2076 (2014).

[30] Hanumegowda, N. M., White, I. M. \& Fan, X. Aqueous mercuric ion detection with microsphere optical ring resonator sensors. Sensors and Actuators, B: Chemical 120, 207-212 (2006). 
[31] Panich, S. et al. Label-Free Pb(II) Whispering Gallery Mode Sensing Using Self- Assembled Glutathione-Modified Gold Nanoparticles on an Optical Microcavity. Anal. Chem 86, 62996306 (2014).

[32] Boening, D. W. Ecological effects, transport, and fate of mercury: A general review. Chemosphere 40, 1335-1351 (2000).

[33] Tchounwou, P. B., Ayensu, W. K., Ninashvili, N. \& Sutton, D. Environmental exposure to mercury and its toxicopathologic implications for public health. Environmental Toxicology 18, 149-175 (2003).

[34] Vallee, B. L. \& Falchuk, K. H. The biochemical basis of zinc physiology. Physiological Reviews 73, 79-118 (1993).

[35] Berg, J. M. \& Shi, Y. The galvanization of biology: a growing appreciation for the roles of zinc. Science 271, 1081-1085 (1996).

[36] Haynes, W. M. CRC Handbook of Chemistry and Physics, 96th Edition, 2015-2016 (Taylor \& Francis Ltd., 2014).

[37] Rex, M., Hernandez, F. E. \& Campiglia, A. D. Pushing the limits of mercury sensors with gold nanorods. Analytical Chemistry 78, 445-451 (2006).

[38] Lee, S. J., Piorek, B. D., Meinhart, C. D. \& Moskovits, M. Photoreduction at a distance: Facile, nonlocal photoreduction of $\mathrm{Ag}$ ions in solution by plasmon-mediated photoemitted electrons. Nano Letters 10, 1329-1334 (2010).

[39] Duval, J. F. L., Huijs, G. K., Threels, W. F., Lyklema, J. \& Van Leeuwen, H. P. Faradaic depolarization in the electrokinetics of the metal-electrolyte solution interface. Journal of Colloid and Interface Science 260, 95-106 (2003).

[40] Barten, D. et al. Double layer of a gold electrode probed by AFM force measurements. Langmuir 19, 1133-1139 (2003).

[41] Pashley, R. M. \& Israelachvili, J. N. A comparison of surface forces and interfacial properties of mica in purified surfactant solutions. Colloids and Surfaces 2, 169-187 (1981).

[42] Pashley, R. M., McGuiggan, P. M., Horn, R. G. \& Ninham, B. W. Forces between bilayers of cetyltrimethylammonium bromide in micellar solutions. Journal of Colloid And Interface Science 126, 569-578 (1988). 